\title{
Prospective Randomized Evaluation of Preoperative Angiotensin-Converting Enzyme Inhibition (PREOP-ACEI)
}

\author{
Jason F. Shiffermiller, MD, MPH', Benjamin J. Monson, MD², Chad W. Vokoun, MD1', Micah W. Beachy, DO', \\ Michael P. Smith, MD1, James N. Sullivan, MD³ , Andrew J. Vasey, MD4, Purnima Guda, PhD², Elizabeth R. Lyden, MS5, \\ Sheila J. Ellis, MD${ }^{3}$, Huiling Pang, MD PhD ${ }^{3}$, Rachel E. Thompson, MD, MPH'
}

\begin{abstract}
${ }^{1}$ Section of Hospital Medicine, University of Nebraska Medical Center, Omaha, Nebraska; ${ }^{2}$ Department of Internal Medicine, University of Nebraska Medical Center, Omaha, Nebraska; ${ }^{3}$ Department of Anesthesiology, University of Nebraska Medical Center, Omaha, Nebraska; ${ }^{4}$ Division of General Internal Medicine, University of Nebraska Medical Center, Omaha, Nebraska; ${ }^{5}$ Department of Biostatistics, University of Nebraska Medical Center, Omaha, Nebraska.
\end{abstract}

BACKGROUND: Intraoperative hypotension is associated with an increased risk of end organ damage and death. The transient preoperative interruption of angiotensinconverting enzyme inhibitor (ACEI) therapy prior to cardiac and vascular surgeries decreases the occurrence of intraoperative hypotension.

OBJECTIVE: We sought to compare the effect of two protocols for preoperative ACEI management on the risk of intraoperative hypotension among patients undergoing noncardiac, nonvascular surgeries.

DESIGN: Prospective, randomized study.

SETTING: Midwestern urban 489-bed academic medical center.

PATIENTS: Patients taking an ACEI for at least six weeks preoperatively were considered for inclusion.

INTERVENTIONS: Randomization of the final preoperative ACEl dose to omission $(n=137)$ or continuation $(n=138)$.

MEASUREMENTS: The primary outcome was intraoperative hypotension, which was defined as any systolic blood pressure (SBP) $<80 \mathrm{~mm} \mathrm{Hg}$. Postoperative hypotensive (SBP $<90 \mathrm{~mm} \mathrm{Hg}$ ) and hypertensive (SBP $>180 \mathrm{~mm} \mathrm{Hg}$ ) episodes were also recorded. Outcomes were compared using Fisher's exact test.

RESULTS: Intraoperative hypotension occurred less frequently in the omission group (76 of 137 [55\%]) than in the continuation group (95 of 138 [69\%]) (RR: 0.81, $95 \% \mathrm{Cl}: 0.67$ to $0.97, \mathrm{P}=.03, \mathrm{NNH} 7.5$ ). Postoperative hypotensive events were also less frequent in the ACEI omission group (RR: $0.49,95 \% \mathrm{Cl}: 0.28$ to $0.86, P=.02$ ) than in the continuation group. However, postoperative hypertensive events were more frequent in the omission group than in the continuation group (RR: $1.95,95 \%$ : $\mathrm{Cl}$ : 1.14 to $3.34, P=.01$ ).

CONCLUSION: The transient preoperative interruption of ACEl therapy is associated with a decreased risk of intraoperative hypotension.

REGISTRATION: ClinicalTrials.gov: NCT01669434. Journal of Hospital Medicine 2018;13:661-667. Published online first July 25, 2018. ( 2018 Society of Hospital Medicine

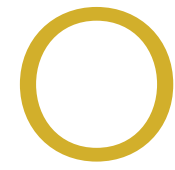

ver seven million surgeries are performed in hospitals in the United States each year. Among these surgeries, approximately $85 \%$ are noncardiac, nonvascular (NCNV) procedures. ${ }^{1,2}$ Although the preoperative use of an angiotensin-converting enzyme inhibitor (ACEI) can be expected in as many as $13 \%$ of these surgeries, ${ }^{3}$ the optimal preoperative ACEI management strategy for patients undergoing NCNV surgeries is poorly understood.

High-quality evidence suggests that renin-angiotensin-aldosterone system (RAAS) inhibitors are associated with intraoperative hypotension among patients undergoing cardiac or vascular surgeries. ${ }^{46}$ Intraoperative hypotension increases the risk of 30-

\footnotetext{
*Address for correspondence: Jason F. Shiffermiller, MD, MPH, $983331 \mathrm{Ne}-$ braska Medical Center, Omaha, NE 68198-3331; Telephone: 402-559-7299; Fax: 402-559-8396; E-mail: jshiffermiller@unmc.edu
}

Received: January 4, 2018; Revised: March 7, 2018; Accepted: March 24, 2018 ๑) 2018 Society of Hospital Medicine DOI 10.12788/jhm.3036 day mortality, and the duration of intraoperative hypotension increases the risk of end organ damage. 8,9 This body of evidence suggests that withholding ACEls prior to cardiac and vascular surgeries is safer than continuing ACEls without interruption.

The evidence concerning perioperative management of ACEls is inconclusive for patients undergoing NCNV procedures. Some studies comparing patients taking or not taking a RAAS inhibitor preoperatively describe negligible differences in the frequency of intraoperative hypotensive episodes or complications. ${ }^{3,10}$ Others have found an increased risk of intraoperative hypotension and associated postoperative adverse events in patients continuing RAAS inhibitors preoperatively. ${ }^{11,12}$ Current guideline discrepancies reflect the uncertainty of the evidence. The guidelines set by the American College of Cardiology and American Heart Association (ACC/AHA) suggest the uninterrupted perioperative continuation of RAAS inhibitors. ${ }^{13}$ The guidelines provided by the European Society of Cardiology and European Society of Anaesthesiology also suggest the continuation of RAAS inhibitors throughout the perioperative period 
for patients with systolic heart failure but recommend transient discontinuation for patients with hypertension. ${ }^{14}$

This randomized study aimed to compare the effect of two practical strategies for preoperative ACEI management on the perioperative blood pressure of patients undergoing NCNV surgery. The two strategies studied were the omission of the final preoperative ACEI dose and the uninterrupted continuation of ACEl therapy. We hypothesized that patients randomized to ACEl omission would experience intraoperative hypotensive episodes less frequently than those randomized to ACEl continuation.

\section{METHODS}

\section{Study Design and Setting}

We performed a prospective randomized controlled trial (ClinicalTrials.gov: NCT01669434). The study was carried out in a preoperative evaluation clinic and its affiliated 489-bed academic medical center. Anesthesiologists and internal medicine physicians work collaboratively in the clinic to assess more than 5,000 patients annually (one-third of the institution's elective surgeries). Patients were randomized 1:1 in block sizes of five and 10 and stratified by age $<65$ and $\geq 65$ years to the omission or continuation of the final preoperative ACEI dose (whether that dose was scheduled for the morning of surgery or the night prior). Preoperative clinicians enrolled patients and subsequently assigned them to intervention groups on the basis of a sequentially numbered list. Patients and healthcare providers were not blinded to allocation status. Intraoperative and postoperative management was provided in accordance with usual care as decided by treatment team.

\section{Participants}

Patients who presented to the preoperative evaluation clinic between May 2015 and November 2016 and who had been taking an ACEl for at least six weeks were eligible for inclusion. Patients taking angiotensin receptor blockers were excluded. Enrollment was limited to patients planning NCNV surgery. Patients planning intrathoracic, major vascular, organ transplant, and oncologic surgery were excluded. Patients undergoing outpatient procedures not requiring an overnight stay in the hospital were also excluded. Patients with preoperative clinic systolic blood pressure (SBP) $<90$ or $\geq 160$ or diastolic blood pressure (DBP) $<60$ or $\geq 95$ were excluded. Patients with moderate to severe or clinically decompensated heart failure (left ventricular ejection fraction $<40 \%$ or New York Heart Association class III or IV) and those with end-stage renal disease requiring dialysis were also excluded. Patients presenting more than once during the accrual period were eligible for the initial surgery only. All participating patients provided written informed consent. This project was approved by the University of Nebraska Medical Center Institutional Review Board.

\section{Data Collection}

Baseline characteristics were recorded by study personnel at the time of enrollment. We measured serum creatinine level at the preoperative visit and on postoperative day one. An automated anesthesia information management system was used to measure intraoperative blood pressures every three minutes. Postoperative blood pressures through discharge were measured by hospital staff per usual care. During postoperative hospitalization, we queried patients about preoperative adherence to allocation. The digital abstraction of data from the electronic medical record was supplemented by chart review when necessary.

\section{Outcomes}

The primary outcome was intraoperative hypotension defined as any $\mathrm{SBP}<80 \mathrm{~mm} \mathrm{Hg}$ occurring from the administration of the first induction agent through transfer to the postanesthesia care unit (PACU). We also examined hypotension during anesthesia induction, which we defined as the 20-minute period following the administration of the first anesthesia induction agent. Episodes of SBP $<80$ were defined as being associated with vasopressor administration when any vasopressor was administered during or within 10 minutes of the episode.

Secondary analyses included postoperative acute kidney injury (AKI), postoperative hypotensive and hypertensive episodes, cardiac events, and mortality. When comparing postoperative day one creatinine levels to preoperative creatinine levels, we used the Acute Kidney Injury Network definition of $\mathrm{AKI}$ as an increase in creatinine of $0.3 \mathrm{mg} / \mathrm{dl}$ or $50 \% .{ }^{15}$ Postoperative hypotension was defined as any SBP $<90 \mathrm{~mm} \mathrm{Hg}$ and postoperative hypertension as any SBP $>180 \mathrm{~mm} \mathrm{Hg}$ occurring after arrival in the PACU. Major adverse cardiac events (MACE) were defined as a composite of acute coronary syndrome, acute heart failure, or new-onset arrhythmia. Discharge from the hospital served as the study endpoint for each patient.

\section{Analysis}

Fisher's exact test was used to compare categorical outcomes between groups. The independent sample t-test or Wilcoxon rank-sum test, as appropriate, was used to compare continuous measures. We selected Fisher's exact test over $\chi^{2}$-test to produce conservative estimates. Patients were maintained in their allocated group as randomized for analytical purposes regardless of adherence to allocation. We performed all analyses using SAS version 9.4 for Windows (SAS institute, Cary, North Carolina).

We estimated that a sample size of 300 patients would achieve $80 \%$ power to detect a difference of 0.17 between the group proportions of 0.33 and 0.50 at a significance level (a) of 0.05 by using a two-sided $z$-test with continuity correction, assuming $15 \%$ loss to follow-up. This estimate allowed for one interim analysis using the $O^{\prime}$ Brien-Fleming spending function truncated at three standard deviations to determine the test boundaries. The monitoring boundary $P$ values associated with the interim analysis were .003 , and the threshold $P$ value for the final analysis was .049 .

\section{RESULTS}

\section{Study Flow}

A total of 453 patients were screened for eligibility. Among these patients, 162 were excluded, and the remaining 291 patients were randomized (Figure 1). Surgery was cancelled in six 


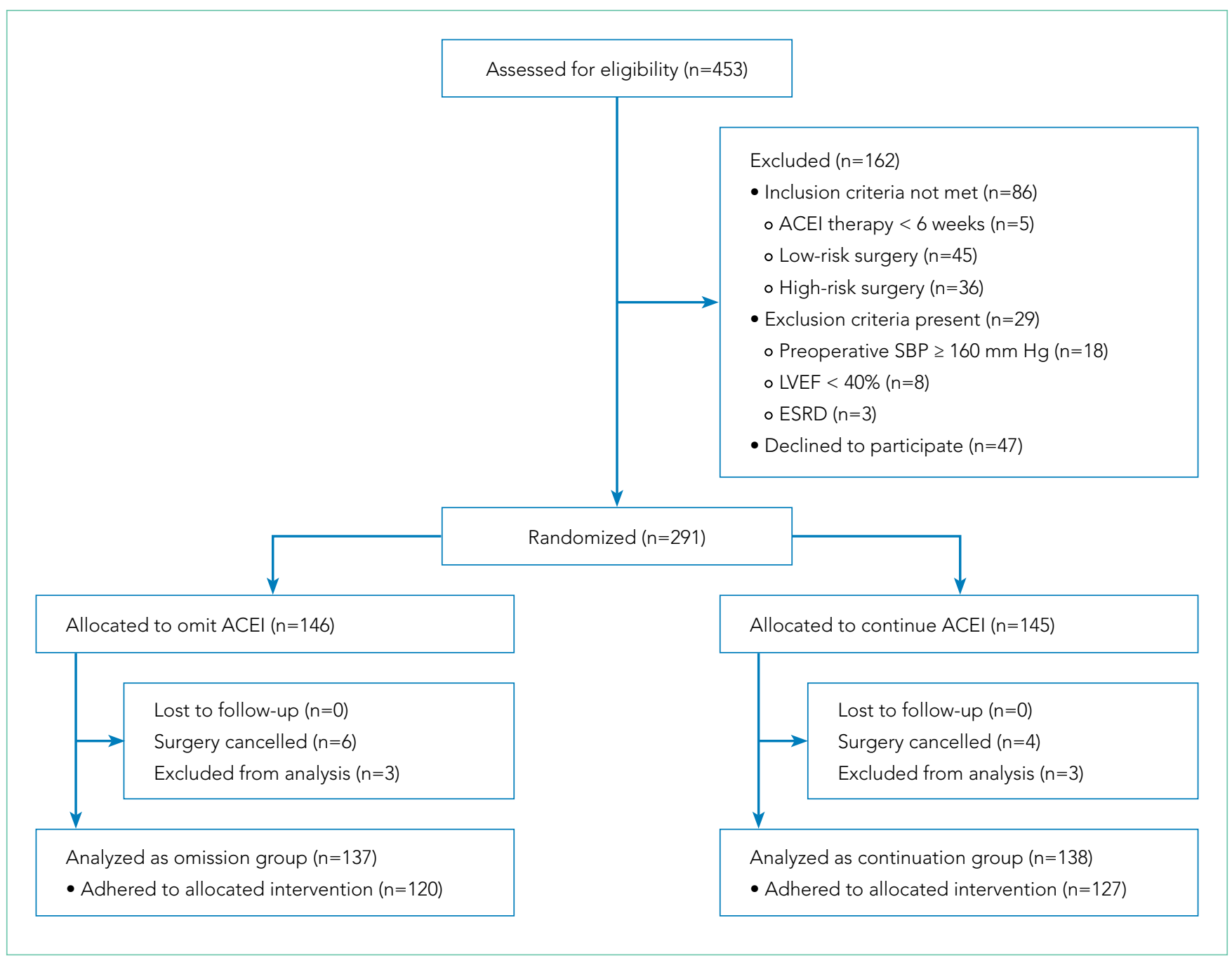

FIG 1. CONSORT diagram.

Abbreviations: ACEI, angiotensin-converting enzyme inhibitor; ESRD, end-stage renal disease; LVEF, left ventricular ejection fraction; SBP, systolic blood pressure.

patients allocated to omission and in four patients allocated to continuation arms, respectively. Moreover, three patients in the omission arm were excluded from the analysis following randomization. Specifically, one was excluded because of early discharge without overnight stay, one was excluded because of withdrawal of consent, and one was excluded because of missing primary outcome data. In addition, three cases in the continuation arm were excluded following randomization because of the preoperative (permanent) discontinuation of ACEI therapy in two cases and discharge without an overnight stay in one case. Finally, 275 patients were included in the analysis: 137 in the ACEl omission group and 138 in the ACEl continuation group. Adherence to allocation was $88 \%$ and $92 \%$ in the omission and continuation groups, respectively.

\section{Baseline Characteristics}

The demographic data of patients allocated to ACEI omission and those allocated to ACEI continuation were similar (Table 1). A large majority of patients in both groups took the ACEI lisinopril. Overall, 187 of 275 (68\%) patients were taking at least one antihypertensive agent, most commonly a diuretic, in addition to an ACEI. SBP measured during the preoperative clinic visit averaged $136.5 \mathrm{~mm} \mathrm{Hg}$ and did not differ significantly between groups $(P=.84)$.

\section{Surgical Variables}

General anesthesia was the most commonly utilized technique, although spinal and regional anesthesia were also represented (Table 1). The majority of cases in both groups were planning for orthopedic and spinal surgery. The method of anesthesia or type of surgery between patients allocated to ACEI omission and those allocated to continuation did not differ $(P=.61$ and $P=.45$ respectively).

\section{Episodes of Intraoperative Hypotension}

Intraoperative SBPs are displayed in Figure 2, and hemodynamic outcomes are summarized in Table 2. Episodes of SBP $<80 \mathrm{~mm} \mathrm{Hg}$ during anesthesia induction were numerically less frequent in the omission group than in the continuation group; the difference between groups, however, was not statistically 
TABLE 1. Patient and Surgical Characteristics by Study Arm

ACEI Omission

$(n=137)$

Patient characteristics

\begin{tabular}{|c|c|c|}
\hline Male sex & $65(47 \%)$ & $68(49 \%)$ \\
\hline Age (years) & $64.0(11.0)$ & $63.7(10.9)$ \\
\hline BMI & $34.4(6.4)$ & $35.0(8.4)$ \\
\hline Caucasian race & $115(84 \%)$ & $125(91 \%)$ \\
\hline \multicolumn{3}{|l|}{ Smoking status } \\
\hline Never & $63(46 \%)$ & $54(39 \%)$ \\
\hline Former & $60(44 \%)$ & $59(43 \%)$ \\
\hline Current & $14(10 \%)$ & $25(18 \%)$ \\
\hline ASA classification & $3(1-4)$ & $3(2-4)$ \\
\hline Revised cardiac risk index & $0(0-3)$ & $0(0-3)$ \\
\hline \multicolumn{3}{|l|}{ Comorbidity } \\
\hline Hypertension & $116(85 \%)$ & $112(81 \%)$ \\
\hline Hyperlipidemia & $78(57 \%)$ & $79(57 \%)$ \\
\hline Coronary artery disease & $18(13 \%)$ & $19(14 \%)$ \\
\hline Diabetes on insulin & $16(12 \%)$ & $19(14 \%)$ \\
\hline COPD & $9(7 \%)$ & $16(12 \%)$ \\
\hline Atrial fibrillation & $10(7 \%)$ & $10(7 \%)$ \\
\hline Congestive heart failure & $7(5 \%)$ & $9(7 \%)$ \\
\hline Preoperative creatinine $>2$ & $1(1 \%)$ & $1(1 \%)$ \\
\hline CVA/TIA & 0 & 0 \\
\hline \multicolumn{3}{|l|}{ ACEI } \\
\hline Lisinopril & $113(82 \%)$ & $109(79 \%)$ \\
\hline Benazepril & $10(7 \%)$ & $11(8 \%)$ \\
\hline Enalapril & $7(5 \%)$ & $7(5 \%)$ \\
\hline Other & $7(5 \%)$ & $11(8 \%)$ \\
\hline Additional antihypertensive & $92(67 \%)$ & $95(69 \%)$ \\
\hline Diuretic & $55(40 \%)$ & $58(42 \%)$ \\
\hline Beta-blocker & $43(31 \%)$ & $51(37 \%)$ \\
\hline Other & $42(31 \%)$ & $34(25 \%)$ \\
\hline NSAID use & $53(39 \%)$ & $71(51 \%)$ \\
\hline Preoperative* systolic BP (mmHg) & $136.6(16.0)$ & $136.3(14.7)$ \\
\hline \multicolumn{3}{|l|}{ Surgical Characteristics } \\
\hline \multicolumn{3}{|l|}{ Anesthesia technique } \\
\hline General & $74(54 \%)$ & $67(49 \%)$ \\
\hline Spinal & $30(22 \%)$ & $36(26 \%)$ \\
\hline Regional & $33(24 \%)$ & $35(25 \%)$ \\
\hline \multicolumn{3}{|l|}{ Type of surgery } \\
\hline Orthopedic & $78(57 \%)$ & $88(64 \%)$ \\
\hline Spine & $29(21 \%)$ & $26(19 \%)$ \\
\hline Bariatric & $7(5 \%)$ & $7(5 \%)$ \\
\hline Otolaryngologic & $5(4 \%)$ & $6(4 \%)$ \\
\hline Other & $18(13 \%)$ & $11(8 \%)$ \\
\hline
\end{tabular}

Categorical variables expressed as number (\%); Continuous variables expressed as mean (SD) when normally distributed and median (range) when not normally distributed. *Preoperativeduring preoperative clinic visit.

Abbreviations: ACEI, angiotensin-converting enzyme inhibitor; ASA, American Society of Anesthesiologists; BMI, Body Mass Index; BP, blood pressure; COPD, chronic obstructive pulmonary

disease; CVA/TIA, history of cerebrovascular accident or transient ischemic attack.

ACEI Continuation

$(n=138)$

$68(49 \%)$

$63.7(10.9)$

$125(91 \%)$

$54(39 \%)$

$25(18 \%)$

$112(81 \%)$

$19(14 \%)$

$19(14 \%)$

0

$109(79 \%)$

$7(5 \%)$

$95(69 \%)$

$34(25 \%)$

$136.6(16.0) \quad 136.3(14.7)$

lic BP (mmHg) 


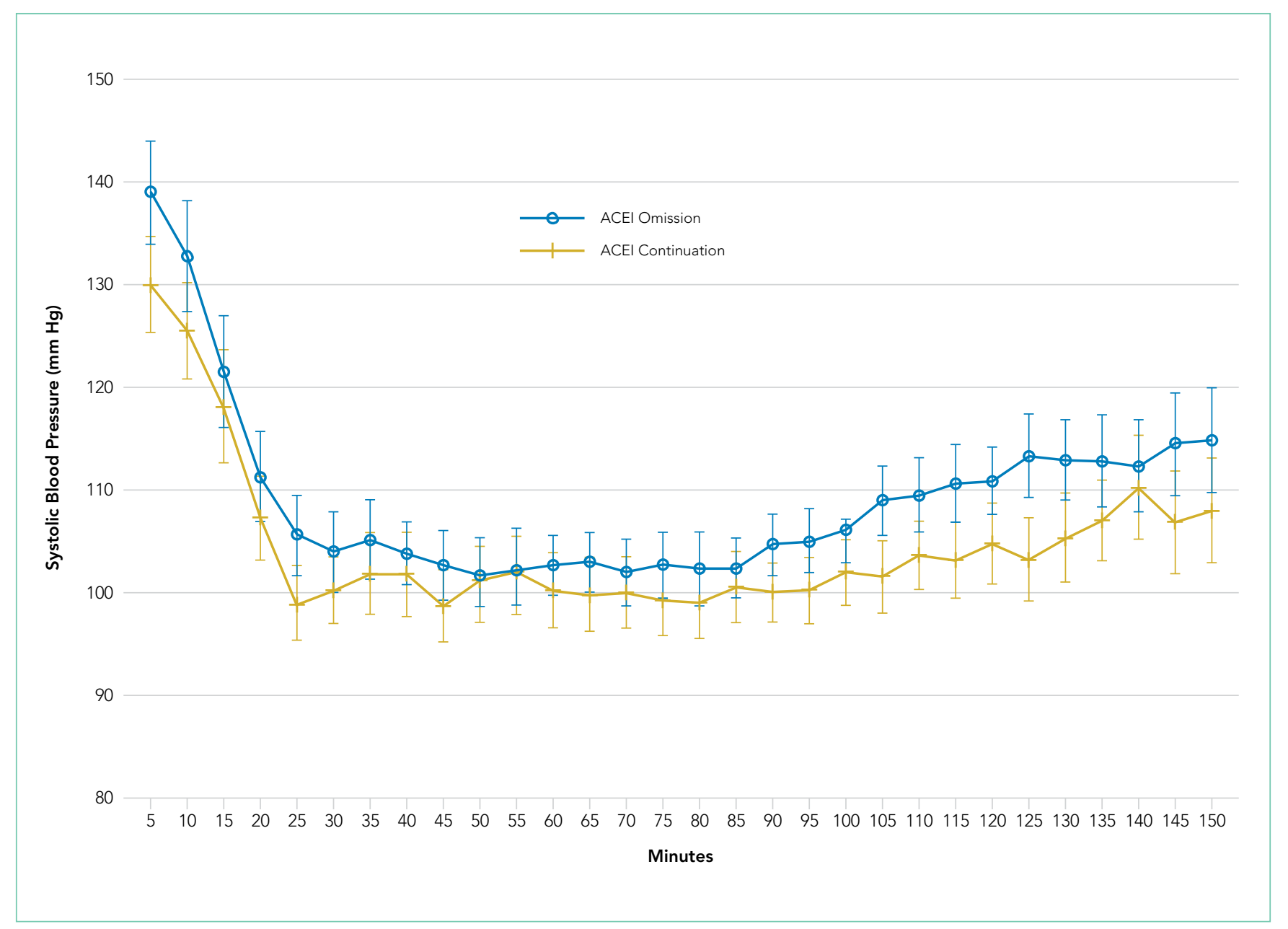

FIG 2. Intraoperative blood pressure. Systolic blood pressures expressed as $5 \mathrm{~min}$ averages. Time $0=$ arrival in operating room. Error bars represent $95 \%$ confidence intervals. Abbreviations: ACEI, angiotensin-converting enzyme inhibitor.

significant (24 of 137 [18\%] vs 38 of 138 [28\%], RR: $0.64,95 \%$ $\mathrm{Cl}: 0.40$ to $1.00, P=.06$ ). The primary outcome, episodes of intraoperative $\mathrm{SBP}<80 \mathrm{~mm} \mathrm{Hg}$, occurred less often in patients allocated to the ACEI omission group than in those allocated to the ACEI continuation group (76 of 137 [55\%] vs 95 of 138 [69\%], RR: $0.81,95 \% \mathrm{Cl}: 0.67$ to $0.97, P=.03$ ). A per-protocol sensitivity analysis of the primary outcome did not substantially alter results (RR: $0.75,95 \% \mathrm{Cl}: 0.61$ to $0.91, P=.003$ ). Among the patients, one was excluded from the sensitivity analysis because of missing data on adherence to allocation. Of the 171 episodes of intraoperative SBP $<80 \mathrm{~mm} \mathrm{Hg}, 149$ were associated with vasopressor administration (61 of 76 [80\%] omission vs 88 of 95 continuation [93\%], RR: $0.87,95 \% \mathrm{Cl}: 0.76$ to 0.98 , $P=.02$ ). Episodes of intraoperative SBP $<80$ associated with vasopressor administration occurred less frequently in patients allocated to the omission group than in those allocated to the continuation group (61/137 [45\%] vs 88/138 [64\%], RR: 0.70, $95 \% \mathrm{Cl}: 0.56-0.87, P<.01)$. Few patients in either group developed severe intraoperative hypotension, which was defined as $\mathrm{SBP}<60 \mathrm{~mm} \mathrm{Hg}$ (6 of 137 [4\%] omission vs 7 of 138 [5\%] continuation, RR: $0.86,95 \% \mathrm{Cl}: 0.30$ to $25.0, P=1.0)$. The number of patients needing to continue ACEl therapy preoperatively to cause one additional episode of harm in the form of intraoperative SBP $<80 \mathrm{~mm} \mathrm{Hg}$ was 7.5 (NNH 7.5).

\section{Duration of Intraoperative Hypotension}

The median cumulative duration of intraoperative SBP $<80$ was two minutes (range 0-41) in patients allocated to the ACEI omission group compared with seven minutes (range 0-214) in those allocated to the continuation group $(P<.01)$. The median cumulative duration of mean arterial pressure $<55 \mathrm{~mm}$ $\mathrm{Hg}$ was also shorter in the omission group (median 0 minutes [range 0-39] vs 3 minutes [range 0-122], $P<.01$ ) than in the continuation group. The duration of surgery did not differ between groups (median 141 minutes [range 77-554] vs $142 \mathrm{~min}$ utes [range 57-665], $P=.97$ ).

\section{Postoperative Outcomes}

RAAS inhibitor therapy was resumed within $48 \mathrm{~h}$ after surgery in 122 of 137 (89\%) patients allocated to the omission group and in 128 of 138 (93\%) patients allocated to the continuation group (RR: $0.96,95 \% \mathrm{Cl}$ : 0.89-1.03, $P=.30$ ).

Patients allocated to the omission group were significantly less likely to experience postoperative hypotension (15 of 137 
TABLE 2. Intraoperative Hemodynamics by Study Arm

\begin{tabular}{|c|c|c|c|}
\hline & $\begin{array}{l}\text { ACEI Omission } \\
\qquad(n=137)\end{array}$ & $\begin{array}{l}\text { ACEI Continuation } \\
\qquad(\mathrm{n}=138)\end{array}$ & $P$ Value \\
\hline Episodes of $\mathrm{SBP}<80 \mathrm{~mm} \mathrm{Hg}$ & $76(55 \%)$ & $95(69 \%)$ & .03 \\
\hline Episodes of SBP $<80 \mathrm{~mm} \mathrm{Hg}$ treated with vasopressor & $61(45 \%)$ & $88(64 \%)$ & $<.01$ \\
\hline Episodes of $\mathrm{SBP}<60 \mathrm{~mm} \mathrm{Hg}$ & $6(4 \%)$ & $7(5 \%)$ & 1.00 \\
\hline Duration of MAP < $55 \mathrm{~mm} \mathrm{Hg}$ (min) & $0(0-39)$ & $3(0-122)$ & $<.01$ \\
\hline
\end{tabular}

Categorical variables expressed as number (percent); Continuous variables expressed as median (range).

Abbreviations: ACEI, angiotensin-converting enzyme inhibitor; MAP, mean arterial pressure; SBP, systolic blood pressure.

[11\%] vs 31 of 138 [22\%], RR: $0.49,95 \% \mathrm{Cl}: 0.28$ to $0.86, P=.02)$ and significantly more likely to experience severe postoperative hypertension (33 of 137 [24\%] vs 17 of 138 [12\%], RR: 1.95, $95 \% \mathrm{Cl}: 1.14$ to $3.34, P=.01)$ than those allocated to the continuation group. The occurrences of postoperative AKI (RR: 0.60, $95 \% \mathrm{Cl}: 0.23$ to $1.60, P=.44$ ) or MACE (RR: $4.03,95 \% \mathrm{Cl}: 0.46$ to $35.59, P=.21$ ) in the omission group did not differ from the continuation group. The two groups exhibited similar PACU recovery time (mean $97.2 \mathrm{~min}$ ) and overall hospital length of stay (mean 3.0 days) $(P=.49$ and $P=.56)$. No episodes of inpatient mortality in either group were observed.

\section{DISCUSSION}

The omission of the final preoperative ACEI dose was associated with a significant reduction in the risk of intraoperative hypotension in patients undergoing NCNV surgery. This result confirmed our hypothesis. Coupled with the knowledge that intraoperative hypotension is associated with an increased risk of complications and mortality, ${ }^{7-9,16}$ this study favors the omission of the final preoperative ACEI dose prior to NCNV surgeries.

Our findings are in agreement with those of previous randomized studies that explored this question ${ }^{4,5}$ and help extend results from cardiac and vascular surgeries to NCNV surgeries. Previous studies on the use of RAAS inhibitors in NCNV surgeries did not employ randomization and yielded mixed results. ${ }^{3,10-12,17}$ A large single-institution study $(n=18,056)$ noted no difference in intraoperative blood pressure between patients taking ACEls and a matched group of non-ACEI users. ${ }^{3}$ More recently, a subgroup analysis of the international VISION study showed that omitting RAAS inhibitors on the day of surgery reduced the risk of intraoperative hypotension. ${ }^{11}$ In that analysis, however, only a small amount of the variability in preoperative RAAS inhibitor management was explainable by modeling known factors, thus allowing for the possibility of unmeasured confounding. Our study, which minimized confounding through randomization, is the first to prospectively compare protocols for patients undergoing NCNV surgery. In contrast to previous studies, the present study was able to report the lack of difference in postoperative RAAS inhibitor administration between study groups. Postoperative RAAS inhibitor management affects complications and mortality. ${ }^{18,19}$
Our present finding that preoperative ACEI management affects postoperative hypotensive and hypertensive events conflicts with some previous findings. ${ }^{11,20}$ However, recent evidence has revealed that postoperative hypotensive episodes are associated with vascular events and mortality. ${ }^{11,21}$ In the context of that evidence, our study lends further support to the omission of the final preoperative ACEI dose. However, we did not detect any decrease in AKI, MACE, or mortality in the ACEl omission group.

This study should be considered in light of its limitations. The pragmatic nature of the study allowed for certain potential biases. Although adherence to allocation was high, the specific ACEl agent taken and the exact timing of the final dose in relation to surgery were not controlled. Anesthetic and postoperative management decisions were made by the treatment team and may have systematically varied given that the treatment team was not blinded to allocation. Furthermore, all outcome data were collected as part of routine care and may not have captured events with great fidelity. Generalizability is limited by the execution of the study at a single academic institution, the preponderance of orthopedic and spine surgeries, and by the negligible representation of ethnicities other than Caucasian. Additionally, recruitment from the preoperative evaluation clinic likely resulted in a patient group with greater comorbidity than the overall population of patients undergoing NCNV surgery. This study was powered for intraoperative hypotension and not postoperative outcomes. Our primary outcome, intraoperative hypotension, is an intermediate measure but one that has well-established associations with adverse outcomes, including mortality. One study showed that sustaining an intraoperative SBP below $70 \mathrm{~mm} \mathrm{Hg}$ for longer than five minutes increased the risk of mortality from less than $1 \%$ to nearly $6 \%{ }^{16} \mathrm{~A}$ large study detected an increase in mortality associated with SBP sustained below $80 \mathrm{~mm} \mathrm{Hg}$ for 10 minutes or longer.? Intraoperative hypotension has also been associated with postoperative AKI and myocardial injury. 8,9,12

Many of the limitations of the current study could be addressed by a large randomized controlled trial of ACEI management prior to NCNV surgeries that examines clinically important endpoints beyond intraoperative hypotension. Several specific aspects of perioperative RAAS inhibitor management 
also deserve further investigation. Our findings may not be generalizable to patients taking ARBs or to patients with congestive heart failure. The preoperative management of ARBs and the preoperative management of RAAS inhibitors in those with congestive heart failure are important areas of focus for future research. Lastly, our finding that preoperative ACEI management decisions can affect postoperative hypotensive and hypertensive events should be substantiated by future research, and any negative consequences of those events should be further explored.

Nonetheless, our study is the largest randomized study of preoperative RAAS inhibition published to date. More than twice as many patients were randomized in this study than in all previous randomized studies combined. ${ }^{4-6}$ To the best of our knowledge, this is also the first randomized study evaluating NCNV surgeries. Finally, our use of a practical ACEI omission protocol based on known pharmacokinetics allows for direct application to clinical practice.

\section{CONCLUSION}

Hypertension is among the most common chronic conditions encountered in patients planning surgery, and ACEls are among the most frequently prescribed antihypertensive medications. This study showed that ACEI continuation is associated with an increased frequency and cumulative duration of intraoperative hypotension. These findings, while at odds with current ACC/AHA guidelines, align with the findings of a meta-analysis on this subject and with recent literature.,311-13,22

\section{Acknowledgments}

The authors wish to thank Miranda M Fricke, MS, PA-C; Tiffany K Hillyard, APRN-FNP; and Barbara Sink, MPAS, PA-C who assisted in the design and conduct of patient enrollment and randomization procedures.

Disclosures: The authors have no relevant financial conflicts of interest to report.

Funding Information: This study was subsidized by a grant from the University of Nebraska Medical Center Research Support Fund. The funding source had no role in the design, conduct, analysis, or reporting of the study.

\section{References}

1. Steiner CA KZ, Moore BJ, Imshaug MC, Pickens G. Surgeries in hospital-based ambulatory surgery and hospital inpatient settings, 2014. Statistical Brief 2017; 1-18. https://www.hcup-us.ahrq.gov/reports/statbriefs/ sb223-Ambulatory-Inpatient-Surgeries-2014.pdf. Accessed August 30, 2017.

2. Rate of all-listed procedures for discharges from short-stay hospitals, by procedure category and age: United States, 2010. National Hospital Discharge Survey 2010; https://www.cdc.gov/nchs/nhds/nhds_tables.htm. Accessed August 30, 2017

3. Turan A, You J, Shiba A, Kurz A, Saager L, Sessler DI. Angiotensin converting enzyme inhibitors are not associated with respiratory complications or mortality after noncardiac surgery. Anesth Analg. 2012;114(3):552-560. doi: 10.1213/ANE.0b013e318241fbaf.

4. Coriat $P$, Richer $C$, Douraki T, et al. Influence of chronic angiotensin-converting enzyme inhibition on anesthetic induction. Anesthesiology. 1994;81:299-307.

5. Pigott DW, Nagle C, Allman K, S. W, D. ER. Effect of omitting regular ACE inhibitor medication before cardiac surgery on haemodynamic variables and vasoactive drug requirements. $\mathrm{Br} J$ Anaesth. 1999;83:715-720. doi: 10.1093/ $\mathrm{bja} / 83.5 .715$
6. Bertrand M, Godet G, Meersschaert K, Brun L, Salcedo E, Coriat P. Should the angiotensin II antagonists be discontinued before surgery? Anesth Analg. 2001;92:26-30

7. Mascha EJ, Yang D, Weiss S, Sessler DI. Intraoperative mean arterial pressure variability and 30-day mortality in patients having noncardiac surgery. Anesthesiology. 2015;123(1):79-91. doi: 10.1097/ALN.00000000000000686.

8. Walsh M, Devereaux PJ, Garg AX, et al. Relationship between intraoperative mean arterial pressure and clinical outcomes after noncardiac surgery: toward an empirical definition of hypotension. Anesthesiology. 2013;119(3):507-515. doi: 10.1097/ALN.0b013e3182a10e26.

9. Salmasi V, Maheshwari K, Yang D, et al. Relationship between intraoperative hypotension, defined by either reduction from baseline or absolute thresholds, and acute kidney and myocardial injury after noncardiac surgery: a retrospective cohort analysis. Anesthesiology. 2017;126(1):47-65. doi: 10.1097/ ALN.0000000000001432

10. Comfere T, Sprung J, Kumar MM, et al. Angiotensin system inhibitors in a general surgical population. Anesth Analg. 2005;100(3):636-644. doi: 10.1213/01.ANE.0000146521.68059.A1

11. Roshanov PS, Rochwerg B, Patel A, et al. Withholding versus continuing angiotensin-converting enzyme inhibitors or angiotensin II receptor blockers before noncardiac surgery: an analysis of the vascular events in noncardiac surgery patlents cohort evaluation prospective cohort. Anesthesiology. 2017;126(1):16-27. doi: 10.1097/ALN.0000000000001404.

12. Nielson E, Hennrikus E, Lehman E, Mets B. Angiotensin axis blockade, hypotension, and acute kidney injury in elective major orthopedic surgery. $J$ Hosp Med. 2014;9(5):283-288. doi: 10.1002/jhm.2155.

13. Fleisher LA, Fleischmann KE, Auerbach AD, et al. 2014 ACC/AHA guideline on perioperative cardiovascular evaluation and management of patients undergoing noncardiac surgery: a report of the American College of Cardiology/American Heart Association Task Force on practice guidelines. J Am Coll Cardiol. 2014;64(22):e77-137. doi: 10.1016/j.jacc.2014.07.944.

14. Kristensen SD, Knuuti J, Saraste A, et al. 2014 ESC/ESA Guidelines on non-cardiac surgery: cardiovascular assessment and management: The Joint Task Force on non-cardiac surgery: cardiovascular assessment and management of the European Society of Cardiology (ESC) and the European Society of Anaesthesiology (ESA). Eur Heart J. 2014;35(35):2383-2431. doi: 10.1093/eurheartj/ ehu282

15. Mehta RL, Kellum JA, Shah SV, et al. Acute Kidney Injury Network: report of an initiative to improve outcomes in acute kidney injury. Crit Care. 2007:11(2):R31. doi: 10.1186/cc5713

16. Monk TG, Bronsert MR, Henderson WG, et al. Association between intraoperative hypotension and hypertension and 30-day postoperative mortality in noncardiac surgery. Anesthesiology. 2015;123(2):307-319. doi: 10.1097/ ALN.0000000000000756.

17. Kheterpal S, Khodaparast O, Shanks A, O'Reilly M, Tremper KK. Chronic angiotensin-converting enzyme inhibitor or angiotensin receptor blocker therapy combined with diuretic therapy is associated with increased episodes of hypotension in noncardiac surgery. $J$ Cardiothorac Vasc Anesth. 2008;22(2):180-186. 10.1053/j.jvca.2007.12.020

18. Lee SM, Takemoto S, Wallace AW. Association between withholding angiotensin receptor blockers in the early postoperative period and 30-day mortality: a cohort study of the veterans affairs healthcare system. Anesthesiology. 2015;123(2):288-306. doi: 10.1097/ALN.0000000000000739.

19. Drenger $B$, Fontes ML, Miao $Y$, et al. Patterns of use of perioperative angiotensin-converting enzyme inhibitors in coronary artery bypass graft surgery with cardiopulmonary bypass: effects on in-hospital morbidity and mortality. Circulation. 2012;126(3):261-269. doi: 10.1161/CIRCULATIONAHA.111.059527.

20. Twersky RS, Goel V, Narayan P, Weedon J. The risk of hypertension after preoperative discontinuation of angiotensin-converting enzyme inhibitors or angiotensin receptor antagonists in ambulatory and same-day admission patients. Anesth Analg. 2014;118(5):938-944. doi: 10.1213/ ANE. 0000000000000076

21. Tan TW, Eslami MH, Kalish JA, et al. The need for treatment of hemodynamic instability following carotid endarterectomy is associated with increased perioperative and 1-year morbidity and mortality. J Vasc Surg. 2014;59(1):1624 e11-12. doi: 10.1053/j.jvca.2014.12.002

22. Rosenman DJ, McDonald FS, Ebbert JO, Erwin PJ, LaBella M, Montori VM. Clinical consequences of withholding versus administering renin-angiotensin-aldosterone system antagonists in the preoperative period. J Hosp Med. 2008;3(4):319-325. doi: 10.1002/jhm.323. 